\title{
Characteristics and outcomes of endoscopically resected colorectal cancers that arose from sessile serrated adenomas and traditional serrated adenomas
}

\author{
Ji Yeon Seo ${ }^{1 *}$, Seung Ho Choi ${ }^{1 *}$, Jaeyoung Chun ${ }^{2}$, Changhyun Lee ${ }^{1}$, Ji Min Choi ${ }^{2}$, Eun Hyo Jin $^{1}$, \\ Sung Wook Hwang ${ }^{2}$, Jong Pil Im², Sang Gyun Kim², Joo Sung Kim ${ }^{1,2}$ \\ ${ }^{I}$ Department of Internal Medicine and Healthcare Research Institute, Healthcare System Gangnam Center, Seoul National University Hospital, \\ ${ }^{2}$ Department of Internal Medicine and Liver Research Institute, Seoul National University College of Medicine, Seoul, Korea
}

\begin{abstract}
Background/Aims: The efficacy and safety of endoscopic resection of colorectal cancer derived from sessile serrated adenomas or traditional serrated adenomas are still unknown. The aims of this study were to verify the characteristics and outcomes of endoscopically resected early colorectal cancers developed from serrated polyps. Methods: Among patients who received endoscopic resection of early colorectal cancers from 2008 to 2011, cancers with documented pre-existing lesions were included. They were classified as adenoma, sessile serrated adenoma, or traditional serrated adenoma according to the baseline lesions. Clinical characteristics, pathologic diagnosis, and outcomes were reviewed. Results: Overall, 208 colorectal cancers detected from 198 patients were included: 198 with adenoma, five with sessile serrated adenoma, and five with traditional serrated adenoma. The sessile serrated adenoma group had a higher prevalence of high-grade dysplasia $(40.0 \% \mathrm{vs.} 25.8 \%, P<0.001)$ than the adenoma group. During follow-up, local recurrence did not occur after endoscopic resection of early colorectal cancers developed from serrated polyps. In contrast, two cases of metachronous recurrence were detected within a short follow-up period. Conclusions: Cautious observation and early endoscopic resection are recommended when colorectal cancer from serrated polyp is suspected. Colorectal cancers from serrated polyp can be treated successfully with endoscopy. (Intest Res 2016;14:270-279)
\end{abstract}

Key Words: Colorectal neoplasms; Colonoscopy; Colonic polyps; Sessile serrated adenoma; Traditional serrated adenoma

\section{INTRODUCTION}

Colorectal cancer (CRC) is the third most common malignancy in the world and the fourth leading cause of cancerrelated death in Korea. ${ }^{1,2}$ Most cases of CRCs originate from adenomatous polyps by an adenoma-carcinoma sequence,

Received July 13, 2015. Revised September 21, 2015

Accepted October 2, 2015.

Correspondence to Changhyun Lee, Department of Internal Medicine and

Healthcare Research Institute, Healthcare System Gangnam Center, Seoul

National University Hospital, 152 Teheran-ro, Gangnam-gu, Seoul 06236,

Korea. Tel: +82-2-2112-5578, Fax: +82-2-2112-5635, E-mail: mdchlee@

gmail.com

*These authors contributed equally to this study.

Financial support: None. Conflict of interest: None. and the incidence and mortality of CRC can be reduced by the early detection and removal of these premalignant lesions. ${ }^{3-5}$ Colonoscopy is a cornerstone of effective CRC prevention, but unfortunately colonoscopy does not prevent all CRCs, especially proximal CRCs. ${ }^{6,7}$

Colon cancers that develop within 5 years of a complete colonoscopy have been termed "interval cancers." Recently, the "serrated neoplasia pathway" of serrated polyps has been identified as an alternative pathway that may account for more than $30 \%$ of interval cancers and $10 \%$ to $20 \%$ of all CRCs. ${ }^{8,9}$ The serrated neoplasia pathway is a sequential change from a hyperplastic polyp (HP) to a sessile serrated adenoma (SSA) that finally develops into a serrated adenocarcinoma. Suggested mechanisms in the serrated neoplasia

\footnotetext{
๑ Copyright 2016. Korean Association for the Study of Intestinal Diseases. All rights reserved.

This is an Open Access article distributed under the terms of the Creative Commons Attribution Non-Commercial License (http://creativecommons.org/licenses/by-nc/4.0)

which permits unrestricted non-commercial use, distribution, and reproduction in any medium, provided the original work is properly cited.
} 
pathway are epigenetic DNA promoter hypermethylations leading to a $\mathrm{CpG}$ island methylator phenotype (CIMP) and microsatellite instability (MSI). CIMP-high and MSI-high are frequently detected in interval cancers. ${ }^{8-12}$ Serrated polyps attract attention as precursors of these interval cancers.

Serrated polyps are classified by the World Health Organization (WHO) into three categories: HP, SSA/polyp, and traditional serrated adenoma (TSA)..$^{10}$ Histologically, a sawtooth appearance of the crypt epithelium is a common feature of serrated polyps. SSAs account for $5 \%-25 \%$ of serrated polyps and are usually found in the proximal colon as they are typically larger than HPs. However, TSAs are rarer than SSAs and are found in the distal colon. ${ }^{10}$

Only a few studies have demonstrated the characteristics of CRCs developed from SSAs (CRC-SSAs) or TSAs (CRCTSAs), and the majority of these papers were case series that were mainly focused on pathologic or molecular characteristics only. ${ }^{13-15}$ There have been no reports that compared the clinical characteristics and outcomes of CRC-SSAs or CRC-TSAs with CRCs developed from conventional adenomas (also called tubular, tubulovillous, or villous adenomas; CRC-adenomas). Furthermore, the effectiveness and safety of endoscopic resection for early CRCs from SSAs or from TSAs are still unknown. The aims of this study were to clarify the clinical characteristics and outcomes of endoscopically resected early CRCs developed from SSAs, TSAs, or adenomas.

\section{METHODS}

\section{Study Population}

A retrospective cohort study was designed. Patients who had endoscopic resection of early CRCs at Seoul National University Hospital (SNUH) from January 2008 to December 2011 were identified. Among them, patients who had early CRCs with pre-existing conventional adenomas, SSAs, or TSAs were included. Patients who had received colectomy previously; patients with hereditary CRCs, such as familial adenomatous polyposis or hereditary non-polyposis CRC; patients with IBD; and patients who did not receive a followup colonoscopy at SNUH were excluded.

\section{Colonoscopy and Bowel Preparation}

Colonoscopy was performed using a standard colonoscopy (Olympus CF H260AL, Olympus, Tokyo, Japan). Generally, $4 \mathrm{~L}$ of polyethylene glycol solution was used for bowel preparation. A colonoscopic polypectomy was not conducted when patients showed inadequate bowel preparation. These patients received additional bowel preparation until it was adequate. During the colonoscopy, all of the resectable early CRCs with lifting signs were resected by either endoscopic mucosal resection (EMR) or endoscopic submucosal dissection (ESD) according to the endoscopist's decision.

\section{Endoscopic Resection of CRC}

The EMR technique is described below. After submucosal injection of saline solution with diluted epinephrine and indigo carmine, circumferential capture of the early CRC with a snare was performed. Polypectomy was executed with a coagulating electrical current. For ESD, after marking and a submucosal injection around the early CRC, cutting was performed with a needle knife followed by submucosal dissection with an insulated-tip electrosurgical knife. Piecemeal EMR or ESD was performed for large CRCs that were difficult to resect by the en bloc method.

\section{Definitions of Variables}

Early CRCs were divided into three groups according to the type of pre-existing adenoma: CRC-adenomas, CRCSSAs, and CRC-TSAs. Locations of CRCs were divided into the proximal and distal colon. The proximal colon was defined as the cecum to the splenic flexure and the distal colon was defined as the descending colon to the rectum. Types of early CRCs were classified as protruded, flat, laterally spreading tumor (LST), or depressed from an endoscopic view according to Kudo's classification. ${ }^{16}$ Early CRCs resected as a whole were termed en bloc, and early CRCs resected in fragments were called piecemeal resections. Numbers of total polyps, adenomas, advanced adenomas, SSAs and/or TSAs, and HPs per patient were counted. Advanced adenoma was defined when more than one of three following characteristics were fulfilled; i.e., size $\geq 1 \mathrm{~cm}$, villous histology, or highgrade dysplasia. ${ }^{17}$

\section{Histological Analysis}

Pathology reports were confirmed by experienced pathologists of SNUH. A pre-existing adenoma of CRC-adenoma was defined as an adenomatous lesion abutting to a CRC, which refers to a precursor lesion of the corresponding CRC. CRC-SSA and CRC-TSA were defined when pre-existing serrated polyps were identified at the edge of a CRC-SSA, CRC- 
TSA, or CRC showed a serrated morphology carcinoma. ${ }^{18}$ A diagnosis of pre-existing serrated polyps was made according to WHO criteria. ${ }^{19}$ SSA was diagnosed when more than three adjacent abnormal crypts and marked dilation of crypts (L or boot shaped) with horizontal bases were detected. Dysplasia of SSA was diagnosed when pseudostratified columnar cells with elongated and hyperchromatic nuclei were detected (conventional dysplasia) ${ }^{10}$ or columnar cells with round nuclei, prominent nucleoli, and scant cytoplasm were noticed (serrated dysplasia). ${ }^{11,13,20}$ TSA was diagnosed when filiform-structure growth and perpendicular formation of crypts from the main crypt (also known as ectopic crypt formation) were observed. TSA with dysplasia was also diagnosed when cytologic dysplasia or serrated dysplasia was detected. Cytologic dysplasia was defined as the conventional dysplasia of SSA. Serrated dysplasia was defined as eosinophilic cytoplasm with large hyperchromatic nuclei. ${ }^{10,12}$ Dysplasia of pre-existing lesions was classified into lowgrade, high-grade, or no dysplasia.

The resection margin was classified as negative, positive, or uncheckable. ${ }^{21}$ Negative referred to the presence of space between the resected margin and the CRC; positive referred to the presence of CRC abutted to the resected margin; and uncheckable was used when the resected margin was blurred by electrical damage or tangential cutting of the specimen. CRC with a positive margin was further classified into positive in the lateral margin, positive in the deep margin, or positive in both the lateral and deep margin. In cases of piecemeal resection, we evaluated all of the fragments of piecemeal-resected specimens and if the main specimen contained all of the cancer tissue with a negative resection margin, it was decided it was a negative resection margin. When the deep margin of the pieces involved cancer tissue, it was considered as a positive resection margin. Uncheckable was selected when neither a negative nor a positive margin was identified or when it was impossible to decide on margin status after reviewing all of the pieces retrieved.

\section{Follow-up Policy}

After endoscopic resection of early CRC, an initial followup colonoscopy was generally performed after 3 months. Subsequent colonoscopies were routinely performed every 6 months for 2 years. When the resection margin was positive after endoscopic resection, an additional operation was recommended. For patients who did not receive additional operations, close follow-up colonoscopies were performed every 3-6 months.

\section{Assessment of Outcomes}

Local recurrence and metachronous recurrence were investigated for the assessment of clinical significance after endoscopic resection of early CRC-SSAs and TSAs. Local recurrence was defined as a newly detected adenoma or carcinoma, located on the scar tissue of the previously resected early CRC at follow-up colonoscopy. A cold biopsy was performed on scar tissue at follow-up colonoscopy to detect local recurrence. Metachronous recurrence was defined as newly detected adenoma or carcinoma other than local recurrence on follow-up colonoscopy. To exclude synchronous recurrence, metachronous recurrence was defined as a new lesion detected after more than 6 months from the initial colonoscopy. ${ }^{22,23}$ When numerous lesions were detected at follow-up colonoscopy, the most advanced lesion was counted for metachronous recurrence. For example, if a new CRC and adenoma were discovered simultaneously at follow-up colonoscopy, the CRC was counted as metachronous recurrence. A single experienced endoscopist (Seo JY) reviewed all images and colonoscopy records and made classifications of local or metachronous recurrence.

Complications of EMR or ESD were also verified. Cases of bleeding and perforation were assessed for complications. Bleeding events were divided into immediate and delayed bleeding on the basis of whether they occurred less than or greater than 24 hours after the procedure, respectively.

\section{Statistical Analysis}

Values were expressed as the median (range) or frequencies (percent). Differences among the three groups were analyzed with a $\chi^{2}$ analysis or Fisher's exact test for nominal variables and the Kruskal-Wallis test for ratio variables. $P$ values less than 0.05 were considered statistically significant. All statistical analyses were performed with SPSS software, version 18.0 (SPSS Inc., Chicago, IL, USA).

\section{Ethics Statement}

The study protocol was approved by the Institutional Review Board of SNUH (IRB No. 1403-092-566) and was conducted in accordance with the Declaration of Helsinki. The written informed consent was waived. 


\section{RESULTS}

\section{Cases of CRCs with Pre-existing SSAs or TSAs}

A total of 208 early CRCs, detected in 198 patients who received endoscopic treatment from January 2008 to December 2011, were analyzed. Among them, there were five CRCSSAs and five CRC-TSAs. All of the CRC-SSAs and CRC-TSAs were a single lesion per patient. The clinical and pathologic features of patients with CRC-SSA and CRC-TSA are shown in Table 1.

Patient 1 received initial diagnostic colonoscopy at an outside hospital for a suspicious lesion detected by PET-CT and was referred to SNUH for endoscopic resection. Therapeutic colonoscopy was performed 2 months after the first colonoscopy. A total of nine polyps were detected, including one CRC-SSA and three SSAs; eight were removed by EMR, and one was removed by ESD. A $3 \mathrm{~cm}$ polyp containing 0.3 $\mathrm{cm}$ of early CRC at the mid transverse colon was removed by piecemeal EMR, and a $2.5 \mathrm{~cm}$ mixed SSA at the distal transverse colon was removed by ESD. However, 5 months after the endoscopic resection, 3.5 and $2.5 \mathrm{~cm}$ SSAs were detected at the follow-up colonoscopy. Prudent reviewing of the images of the first colonoscopy revealed two $1.0 \mathrm{~cm}$ LSTs at the same site of the colon as seen on the follow-up colonoscopy. These 2 large missed SSAs were not included for metachronous recurrence. The PET-CT image, endoscopic capture image, and histologic pictures of the CRC-SSA are displayed in Fig. 1.

The two CRC-SSAs (patients 1 and 2) were resected by piecemeal resection but the resection margins were negative and local recurrence did not occur. One patient (patient 3) with en bloc resection had involvement of the lateral margin by carcinoma but local recurrence was absent during the follow-up period.

Patient 7 received colonoscopy for a positive stool hemoglobin test. She had six polyps at the initial colonoscopy; one was an intramucosal adenocarcinoma with a baseline TSA, three were serrated polyps, and two were low grade tubular adenomas. A sufficient resection margin was achieved for the early CRC; i.e., $0.9 \mathrm{~cm}$ for both the lateral and deep resection margin. Three months later, a $0.5 \mathrm{~cm}$ adenoma in the sigmoid colon, which was the same segment that the previous early CRC arose in, was detected at a subsequent colonoscopy. Because scar tissue was absent around this adenoma and the pathologic type was different, this adenoma could be a missed lesion rather than local recurrence.

Nine of ten patients were checked with CT scan or PET-





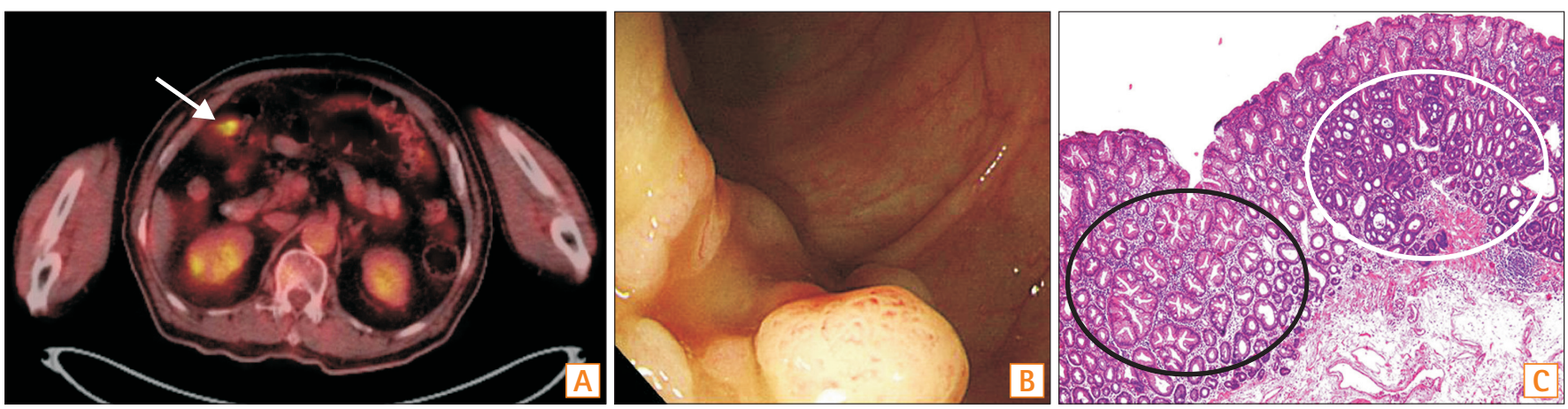

Fig. 1. Colonoscopic and histologic pictures of polyps in patient 1. (A) PET-CT image shows signal intensity in the mid transverse colon (arrow). (B) Colonoscopic features of colorectal cancer (CRC) developed from sessile serrated adenoma (SSA). (C) Histologic features of CRC (white circle) developed from SSA (black circle) $(H C t E, \times 100)$.

CT for verification of lymph node or distant metastasis and none of the patients had lymph node or distant metastasis. Patient 2, who had submucosal invasion of the CRC, did not undergo additional work-up or surgery because of underlying cardiac disease and old age. Three patients (patients 1, 4, and 6) received PET-CT before endoscopic resection of CRCs. Patients 1 and 6 showed a high standardized uptake value (SUV, 9.0 and 13.5, respectively), but there was no signal intensity on the PET-CT of patient 4.

\section{Baseline Characteristics of Patients and Endoscopic Features of CRCs}

Baseline characteristics of the patients and endoscopic features of the early CRCs according to pre-existing lesions are displayed in Table 2 . The median age of the patients was 63 (30-82) years in patients with CRC-adenomas, 70 (55-80) years in patients with CRC-SSAs, and 64 (40-73) years in patients with CRC-TSAs, which were not significantly different among the three groups. Patients with CRC-SSAs and with CRC-TSAs were predominantly women $(n=3,60.0 \%$ for each), but CRC-adenomas occurred less frequently in women ( $\mathrm{n}=62,33.0 \%)$. The numbers of adenomas, advanced adenomas, HPs, and accompanying SSAs and/or TSAs per patient were not significantly different among the three groups. Tumor markers, such as CEA, CA19-9, and size of the early CRCs, were not significantly different according to the type of pre-existing lesions. CRC-adenomas ( $\mathrm{n}=154,77.8 \%)$ and CRC-TSAs $(n=4,80.0 \%)$ tended to localize to the distal colon, and CRC-SSAs tended to be located in the proximal colon ( $\mathrm{n}=3,60.0 \%)$. The protruded type of early CRCs were the most common in CRC-TSAs $(\mathrm{n}=4,80.0 \%)$. In contrast, proportions of the protruded type and LST were the same in CRC-SSAs ( $\mathrm{n}=2,40.0 \%)$. All of the early CRCs with underly- ing SSAs and TSAs received EMR. En bloc resection was performed for 146 (73.7\%) CRC-adenomas, 3 (60.0\%) CRCSSAs, and all CRC-TSAs $(\mathrm{n}=5)$.

\section{Complications of Endoscopic Resection Procedures}

Complications of endoscopic resection procedures are also shown in Table 2. In terms of complications, there was only one micro-perforation (20.0\%) case in a patient with CRC-SSA (patient 1). After the procedure, abdominal pain and fever developed and a chest radiograph showed intraabdominal free air. Fortunately, the patient recovered after medical treatment. Complications did not occur in patients with CRC-TSAs. Bleeding did not occur after endoscopic resection of CRC-SSAs or CRC-TSAs.

\section{Pathologic Characteristics of CRCs}

The pathology results of early CRCs according to the preexisting lesions are shown in Table 3. The resection margin was negative in all CRC-TSAs. In contrast, among five CRCSSAs in the CRC-SSA group, 3 (60.0\%) had a negative margin, $1(20.0 \%)$ had a positive margin, and $1(20.0 \%)$ had an uncheckable margin. Those results seemed to indicate a worse outcome than CRC-adenomas, however these differences were not significant $(P=0.573)$. An additional operation for a positive resection margin was performed for 9 (4.5\%) CRC-adenomas. None of the patients with CRC-SSAs received additional treatment.

Two CRC-SSAs (40.0\%) had high-grade dysplasia, which was a higher rate than that in CRC-adenomas $(n=51,25.8 \%)$. Only two CRC-TSAs (40.0\%) had low-grade dysplasia and the remaining three CRC-TSAs (60.0\%) showed an absence of dysplasia. These differences among groups were signifi- 
Table 2. Baseline Characteristics of Patients and Colorectal Cancers (CRCs) According to Pre-existing Adenoma, Sessile Serrated Adenoma (SSA), or Traditional Serrated Adenoma (TSA)

\begin{tabular}{|c|c|c|c|c|}
\hline Variable & CRC-Adenoma & CRC-SSA & CRC-TSA & $P$-value \\
\hline Total no. of patients & 188 & 5 & 5 & \\
\hline Total no. of CRCs & 198 & 5 & 5 & \\
\hline Age & $63(30-82)$ & $70(55-80)$ & 64 (40-73) & 0.521 \\
\hline Sex & & & & 0.215 \\
\hline Male & $126(67.0)$ & $2(40.0)$ & $2(40.0)$ & \\
\hline Female & $62(33.0)$ & $3(60.0)$ & $3(60.0)$ & \\
\hline Comorbidity & & & & 0.369 \\
\hline Cardiopulmonary disease & $13(6.9)$ & $2(40.0)$ & $1(20.0)$ & \\
\hline Chronic kidney disease & $2(1.1)$ & 0 & 0 & \\
\hline Chronic liver disease & $1(0.5)$ & 0 & 0 & \\
\hline Cancer (except CRC) & $27(14.4)$ & 0 & $1(20.0)$ & \\
\hline None & $145(77.1)$ & $3(60.0)$ & $3(60.0)$ & \\
\hline No. of adenomas ${ }^{a}$ & $1(0-16)$ & $0(0-2)$ & $1(0-2)$ & 0.298 \\
\hline No. of advanced adenomas ${ }^{a}$ & $0(0-7)$ & $0(0-2)$ & 0 & 0.377 \\
\hline No. of SSA/TSAs ${ }^{a}$ & $0(0-3)$ & $0(0-3)$ & 0 & 0.262 \\
\hline No. of hyperplastic polyps ${ }^{a}$ & $0(0-3)$ & $0(0-2)$ & $0(0-3)$ & 0.593 \\
\hline CEA (ng/ml) & $1.7(0.8-5.7)$ & $1.8(1.7-1.8)$ & $2.5(0.5-2.8)$ & 0.822 \\
\hline CA19-9 (U/ml) & $7.7(1.0-54.0)$ & $9.1(1.6-16.6)$ & $6.6^{c}$ & 0.915 \\
\hline Size $(\mathrm{cm})$ & $0.5(0.1-3.7)$ & $0.5(0.3-1.3)$ & $0.8(0.6-1.1)$ & 0.362 \\
\hline Site $^{b}$ & & & & 0.139 \\
\hline Proximal colon & $44(22.2)$ & $3(60.0)$ & $1(20.0)$ & \\
\hline Distal colon & $154(77.8)$ & $2(40.0)$ & $4(80.0)$ & \\
\hline Type & & & & 0.086 \\
\hline Protruded & 166 (83.8) & $2(40.0)$ & $4(80.0)$ & \\
\hline Flat & $6(3.0)$ & $1(20.0)$ & 0 & \\
\hline Laterally spreading & $26(13.1)$ & $2(40.0)$ & $1(20.0)$ & \\
\hline Depressed & 0 & 0 & 0 & \\
\hline Endoscopic resection & & & & 0.879 \\
\hline EMR & $193(97.5)$ & $5(100.0)$ & $5(100.0)$ & \\
\hline ESD & $5(2.5)$ & 0 & 0 & \\
\hline Type of resection & & & & 0.321 \\
\hline En bloc & $146(73.7)$ & $3(60.0)$ & $5(100.0)$ & \\
\hline Piecemeal & $52(26.3)$ & $2(40.0)$ & 0 & \\
\hline \multicolumn{5}{|l|}{ Complication } \\
\hline Bleeding $\leq 24 \mathrm{~h}$ & $1(0.5)$ & 0 & 0 & \\
\hline Bleeding $>24 \mathrm{~h}$ & $5(2.5)$ & 0 & 0 & \\
\hline Perforation & $2(1.0)$ & $1(20.0)$ & 0 & \\
\hline
\end{tabular}

Values are presented as median (range) or $n(\%)$.

${ }^{a}$ Number of lesions per patient.



'CA19-9 was performed for only one patient in the CRC-TSA group.

EMR, endoscopic mucosal resection; ESD, endoscopic submucosal dissection. 
Table 3. Pathology of Colorectal Cancers (CRCS) According to Pre-existing Adenoma, Sessile Serrated Adenoma (SSA), or Traditional Serrated Adenoma (TSA)

\begin{tabular}{|c|c|c|c|c|}
\hline Variable & CRC-Adenoma & CRC-SSA & CRC-TSA & $P$-value \\
\hline Resection margin & & & & 0.573 \\
\hline Negative & 164 (82.8) & $3(60.0)$ & $5(100.0)$ & \\
\hline Positive $^{a}$ & $19(9.6)$ & $1(20.0)$ & 0 & \\
\hline Lateral margin & $6(3.0)$ & $1(20.0)$ & 0 & \\
\hline Deep margin & $7(3.5)$ & 0 & 0 & \\
\hline Both lateral and deep margin & $6(3.0)$ & 0 & 0 & \\
\hline Uncheckable & $15(7.6)$ & $1(20.0)$ & 0 & \\
\hline Dysplasia of pre-existing adenoma & & & & $<0.001$ \\
\hline No dysplasia & 0 & $2(40.0)$ & $3(60.0)$ & \\
\hline Low-grade dysplasia & $147(74.2)$ & $1(20.0)$ & $2(40.0)$ & \\
\hline High-grade dysplasia & $51(25.8)$ & $2(40.0)$ & 0 & \\
\hline Depth of invasion & & & & 0.253 \\
\hline Intramucosal & $133(67.2)$ & $4(80.0)$ & $5(100.0)$ & \\
\hline Submucosal & $65(32.8)$ & $1(20.0)$ & 0 & \\
\hline Angiolymphatic invasion & & & & 0.806 \\
\hline Yes & $8(4.1)$ & 0 & 0 & \\
\hline No & 185 (95.9) & $5(100.0)$ & $5(100.0)$ & \\
\hline \multicolumn{5}{|l|}{ Venous invasion } \\
\hline Yes & 0 & 0 & 0 & \\
\hline No & 193 (100.0) & $5(100.0)$ & $5(100.0)$ & \\
\hline \multicolumn{5}{|l|}{ Perineural invasion } \\
\hline Yes & 0 & 0 & 0 & \\
\hline No & 193 (100.0) & $5(100.0)$ & $5(100.0)$ & \\
\hline
\end{tabular}

Values are presented as $n(\%)$.

${ }^{a}$ Positive resection margin refers to the presence of CRC abutted to the resected margin.

cant $(P<0.001)$. The CRC-SSA and CRC-TSA groups tended to have a lower incidence of submucosal invasion $(\mathrm{n}=1$, $20.0 \%$ in the CRC-SSA group and $\mathrm{n}=0$ in the CRC-TSA group, $P=0.253)$. Neither the CRC-SSA group nor the CRC-TSA group had angiolymphatic, venous, or perineural invasion of cancer. On the contrary, 8 patients $(4.1 \%)$ in the CRCadenoma group had angiolymphatic invasion.

\section{Clinical Outcomes of CRCs}

Clinical outcomes of early CRCs according to baseline lesions are displayed in Table 4 . The median follow-up duration was 23.5 (2.6-71.1), 11.8 (4.7-27.8), and 20.8 (6.1-30.6) months in patients in the CRC-adenoma group, the CRCSSA group, and the CRC-TSA group, respectively. There were 15 (7.5\%) local recurrences in the CRC-adenoma group; 6 $(3.0 \%)$ were recurrences of CRCs and $9(4.5 \%)$ were recur- rences of adenomas. There was no local recurrence of either CRC or adenoma after endoscopic resection in the CRC-SSA group or the CRC-TSA group. For metachronous recurrence, $2(1.0 \%)$ cases had new CRCs, and 21 (10.6\%) cases had new advanced adenomas in the CRC-adenoma group, while neither metachronous CRCs nor advanced adenomas were detected in the CRC-SSA group or the CRC-TSA group. A single case of metachronous adenomas $(10.0 \%)$ was detected in each of the CRC-SSA and CRC-TSA groups. Two adenomas were detected after 11.8 months follow-up in patient 2 and similarly two adenomas were detected after 6.8 months from initial colonoscopy in patient 9 .

\section{DISCUSSION}

We investigated the clinical characteristics and outcomes of endoscopically resected early CRCs. The CRC-SSA group 
Table 4. Clinical Outcomes of Colorectal Cancers (CRCS) According to Pre-existing Adenoma, Sessile Serrated Adenoma (SSA), or Traditional Serrated Adenoma (TSA)

\begin{tabular}{lccc}
\hline \multicolumn{1}{c}{ Variable } & CRC-Adenoma & CRC-SSA & CRC-TSA \\
\hline Follow-up duration (mo) & $23.5(2.6-71.1)$ & $11.8(4.7-27.8)$ & \\
Local recurrence & & & 0 \\
CRC & $6(3.0)$ & 0 & 0 \\
Adenoma & $9(4.5)$ & - & - \\
Time to local recurrence (mo) & $14.0(3.1-38.5)$ & & 0 \\
Metachronous recurrence & & 0 & 0 \\
CRC & $2(1.0)$ & 0 & $1(10.0)$ \\
Advanced adenoma & $21(10.6)$ & $1(10.0)$ & 0 \\
Adenoma & $70(35.4)$ & 0 & 0 \\
SSA & $3(1.5)$ & 0 & 11.8 \\
TSA & 0 & 6.8 & 0 \\
Time to metachronous recurrence (mo) & $14.0(6.1-67.3)$ & & 0 \\
\hline
\end{tabular}

Values are presented as median (range) or $n(\%)$.

had a higher prevalence of high-grade dysplasia in preexisting lesions $(P<0.001)$ than those with CRC-adenomas. All early CRCs with baseline SSA/TSAs were successfully treated by EMR and there was no local recurrence after resection of CRC-SSA or CRC-TSA. Metachronous recurrence of adenomas occurred in a single case in each of the CRCSSA and CRC-TSA groups. To our knowledge, this is the first study to evaluate clinical characteristics and follow-up outcomes of endoscopic resection of CRC-SSA and CRC-TSA compared with CRC-adenoma.

In previous reports, synchronous and metachronous lesions were frequent when serrated polyp was detected. ${ }^{24,25} \mathrm{In}$ this study, the number of synchronous lesions was not significantly different and metachronous recurrences could not be compared among the three groups because of the small number of cases in the CRC-SSA and CRC-TSA groups. However, upon reviewing our 10 cases of CRC-SSAs and CRC-TSAs, new lesions were discovered in two cases after 6 months. As with patient 1, SSAs are frequently missed because SSAs tend to localize to the proximal colon, and they usually have a flat shape and are covered with mucus. ${ }^{26,27}$ Although various techniques were introduced to improve the detection of SSA, the endoscopist's effort to detect and remove SSAs was the most important factor. ${ }^{28}$ Considering that numerous new lesions were detected within a short period, careful examination to find synchronous lesions is needed, especially if CRC developed from a serrated polyp is suspected during colonoscopy.

In this study, the CRC-SSA group showed a higher rate of high-grade dysplasia than those in the CRC-adenoma group. These findings are considered strong evidence of the "serrated neoplasia pathway" because the progression of dysplasia in a sessile serrated polyp is a key step in becoming CRC through the "serrated neoplasia pathway." However, the CRC-TSA group showed a higher rate of absence of dysplasia $(P<0.001)$, and this finding suggests a possible different pathway of CRC development from TSA.

Previously, Goldstein reported pathologic features of eight cases in which serrated polyps developed into invasive adenocarcinoma or high-grade dysplasia, and six cases of these small-sized adenocarcinomas invaded into the submucosa. ${ }^{13}$ Ban et al. also reported that five CRCs among eight cases of CRC-SSAs invaded into the submucosa or muscularis propria layer. ${ }^{15}$ These results suggest that serrated adenocarcinomas might invade downward rather than laterally early in their carcinomatous phase.$^{13}$ However, among 10 cases of CRCs from serrated polyps in our study, only one case of CRC-SSA invaded into the submucosa, and the depth of invasion and size of the CRC was not different between CRCSSAs and CRC-adenomas. This discrepancy may be a possible selection bias because only patients with endoscopically resected CRCs were enrolled in this study, and these lifted lesions are generally considered as a lesion confined to the mucosa.

In this study, patient 1 is an interesting case for displaying the natural course of SSAs. Two $1 \mathrm{~cm}$ SSAs detected at an outside colonoscopy rapidly grew to $3.5 \mathrm{~cm}$ and $2.5 \mathrm{~cm}$ (Fig. 1) during 7 months. This finding may demonstrate the 
aggressive nature of SSAs. Furthermore, he had five serrated polyps proximal to the sigmoid colon and four of them were larger than $1 \mathrm{~cm}$. These conditions satisfied the diagnostic criteria of serrated polyposis syndrome (SPS). ${ }^{29}$ The SPS is associated with a high prevalence of accompanying CRCs and a 1-year surveillance interval for SPS patients is recommended.

In this study, one of two cases in the CRC-SSA group and one case in the CRC-TSA group who received PET-CT had high SUVs. In our previous PET-CT study, the sensitivity of PET-CT for CRC was $88.1 \%$ and for advanced adenoma it was $54.4 \%{ }^{30}$ However, there has been no previous report about PET-CT results related to serrated polyps or serrated adenocarcinomas. Considering that most of these lesions are difficult to discover and easily missed on colonoscopy, the effectiveness of PET-CT for detecting serrated lesions would be an interesting investigation.

There are important advantages to this study. Because serrated adenocarcinoma is rare, only a few case series were published with a small number of cases, and these were mainly focused on pathologic features. We elucidated the clinical characteristics of early CRCs from SSAs or TSAs and compared them with early CRCs from conventional adenomas. Furthermore, we demonstrated the outcome, safety, and effectiveness of endoscopic resection for early CRCs derived from SSAs or TSAs. Finally, local recurrence did not occur after endoscopic resection of CRC-SSA and CRC-TSA. Therefore, a colonoscopy surveillance program similar to that used for CRC-adenoma could be considered after endoscopic resection of CRC-SSA or CRC-TSA.

However, there are several limitations to our study. First, this was a retrospective study, and it is therefore subject to drawbacks that are inherent in retrospective analysis, such as the potential for missing cases and selection bias. Second, CRC-SSAs and CRC-TSAs are rare diseases; therefore, the total number of patients with CRC-SSAs or CRC-TSAs was not enough to perform statistical analyses and interpretation. Third, molecular pathology was not verified in our study. Finally, because our study enrolled only patients with endoscopically resected early CRCs, only patients with relatively early CRCs were enrolled and this may be a selection bias.

In conclusion, cautious observation of synchronous lesions and early endoscopic resection of SSA/TSA are necessary for decreasing the number of interval cancers. CRCs developed from serrated polyps can be treated by endoscopy successfully.

\section{REFERENCES}

1. Siegel R, Naishadham D, Jemal A. Cancer statistics, 2012. CA Cancer J Clin 2012;62:10-29.

2. Park HC, Shin A, Kim BW, et al. Data on the characteristics and the survival of Korean patients with colorectal cancer from the Korea central cancer registry. Ann Coloproctol 2013;29:144149.

3. Burt RW. Colon cancer screening. Gastroenterology 2000;119: 837-853.

4. Zauber AG, Winawer SJ, O'Brien MJ, et al. Colonoscopic polypectomy and long-term prevention of colorectal-cancer deaths. N Engl J Med 2012;366:687-696.

5. Gupta AK, Melton LJ 3rd, Petersen GM, et al. Changing trends in the incidence, stage, survival, and screen-detection of colorectal cancer: a population-based study. Clin Gastroenterol Hepatol 2005;3:150-158.

6. Farrar WD, Sawhney MS, Nelson DB, Lederle FA, Bond JH Colorectal cancers found after a complete colonoscopy. Clin Gastroenterol Hepatol 2006;4:1259-1264.

7. Lieberman DA, Holub J, Eisen G, Kraemer D, Morris CD. Utilization of colonoscopy in the United States: results from a national consortium. Gastrointest Endosc 2005;62:875-883.

8. Leggett B, Whitehall V. Role of the serrated pathway in colorectal cancer pathogenesis. Gastroenterology 2010;138:2088-2100.

9. Terdiman JP, McQuaid KR. Surveillance guidelines should be updated to recognize the importance of serrated polyps. Gastroenterology 2010;139:1444-1447.

10. Rex DK, Ahnen DJ, Baron JA, et al. Serrated lesions of the colorectum: review and recommendations from an expert panel. Am J Gastroenterol 2012;107:1315-1329.

11. Snover DC. Update on the serrated pathway to colorectal carcinoma. Hum Pathol 2011;42:1-10.

12. Sweetser S, Smyrk TC, Sinicrope FA. Serrated colon polyps as precursors to colorectal cancer. Clin Gastroenterol Hepatol 2013;11:760-767.

13. Goldstein NS. Small colonic microsatellite unstable adenocarcinomas and high-grade epithelial dysplasias in sessile serrated adenoma polypectomy specimens: a study of eight cases. Am J Clin Pathol 2006;125:132-145.

14. Xu S, Wang L, Yang G, et al. Clinicopathological observations of colorectal serrated lesions associated with invasive carcinoma and high-grade intraepithelial neoplasm. Exp Ther Med 2013;6:1113-1120.

15. Ban S, Mitomi H, Horiguchi H, Sato H, Shimizu M. Adenocarcinoma arising in small sessile serrated adenoma/polyp (SSA/ P) of the colon: clinicopathological study of eight lesions. Pathol Int 2014;64:123-132. 
16. Kudo S, Kashida H, Tamura T, et al. Colonoscopic diagnosis and management of nonpolypoid early colorectal cancer. World J Surg 2000;24:1081-1090.

17. Seo JY, Chun J, Lee C, et al. Novel risk stratification for recurrence after endoscopic resection of advanced colorectal adenoma. Gastrointest Endosc 2015;81:655-664.

18. Bettington M, Walker N, Clouston A, Brown I, Leggett B, Whitehall V. The serrated pathway to colorectal carcinoma: current concepts and challenges. Histopathology 2013;62:367-386.

19. Coindre JM. New WHO classification of tumours of soft tissue and bone. Ann Pathol 2012;32 (5 Suppl):S115-S116.

20. Torlakovic E, Skovlund E, Snover DC, Torlakovic G, Nesland JM. Morphologic reappraisal of serrated colorectal polyps. Am J Surg Pathol 2003;27:65-81.

21. Choi JM, Lee C, Park JH, et al. Complete resection of colorectal adenomas: what are the important factors in fellow training? Dig Dis Sci 2015;60:1579-1588.

22. Lieberman DA, Weiss DG, Harford WV, et al. Five-year colon surveillance after screening colonoscopy. Gastroenterology 2007;133:1077-1085.

23. Martínez ME, Baron JA, Lieberman DA, et al. A pooled analysis of advanced colorectal neoplasia diagnoses after colonoscopic polypectomy. Gastroenterology 2009;136:832-841.
24. Hiraoka S, Kato J, Fujiki S, et al. The presence of large serrated polyps increases risk for colorectal cancer. Gastroenterology 2010;139:1503-1510.

25. Lu FI, van Niekerk de W, Owen D, Tha SP, Turbin DA, Webber DL. Longitudinal outcome study of sessile serrated adenomas of the colorectum: an increased risk for subsequent right-sided colorectal carcinoma. Am J Surg Pathol 2010;34:927-934.

26. Teriaky A, Driman DK, Chande N. Outcomes of a 5-year followup of patients with sessile serrated adenomas. Scand J Gastroenterol 2012;47:178-183.

27. Rustagi T, Rangasamy P, Myers M, et al. Sessile serrated adenomas in the proximal colon are likely to be flat, large and occur in smokers. World J Gastroenterol 2013;19:5271-5277.

28. Dik VK, Moons LM, Siersema PD. Endoscopic innovations to increase the adenoma detection rate during colonoscopy. World J Gastroenterol 2014;20:2200-2211.

29. Aust DE, Baretton GB; Members of the Working Group GIPathology of the German Society of Pathology. Serrated polyps of the colon and rectum (hyperplastic polyps, sessile serrated adenomas, traditional serrated adenomas, and mixed polyps)proposal for diagnostic criteria. Virchows Arch 2010;457:291297.

30. Lee C, Koh SJ, Kim JW, et al. Incidental colonic 18F-fluorodeoxyglucose uptake: do we need colonoscopy for patients with focal uptake confined to the left-sided colon? Dig Dis Sci 2013;58:229-235. 